\title{
BMJ Open Registry of Older South Australians (ROSA): framework and plan
}

\author{
Maria C Inacio, ${ }^{1,2}$ Sarah Catherine Elizabeth Bray, ${ }^{01,2,3}$ Craig Whitehead, ${ }^{4,5}$ \\ Megan Corlis, ${ }^{6}$ Renuka Visvanathan, ${ }^{7,8}$ Keith Evans, ${ }^{9}$ Elizabeth C Griffith, ${ }^{10}$ \\ Steve L Wesseling ${ }^{10}$
}

To cite: Inacio MC, Bray SCE, Whitehead C, et al. Registry of Older South Australians (ROSA): framework and plan. BMJ Open 2019;9:e026319. doi:10.1136/ bmjopen-2018-026319

- Prepublication history for this paper is available online. To view these files, please visit the journal online (http://dx.doi. org/10.1136/bmjopen-2018026319).

Received 27 August 2018 Revised 25 January 2019 Accepted 10 April 2019

\section{Check for updates}

(c) Author(s) (or their employer(s)) 2019. Re-use permitted under CC BY-NC. No commercial re-use. See rights and permissions. Published by BMJ.

For numbered affiliations see end of article.

Correspondence to Dr. Sarah Catherine

Elizabeth Bray;

Sarah.Bray@sahmri.com

\section{ABSTRACT}

Introduction Australia's ageing population puts significant demands on the aged care and healthcare sectors. To monitor the provision of aged care and healthcare services to older people, each government body has an individual data collection system. Together these systems can be the basis for creating the evidence necessary to support future allocation of resources for our ageing community. The Registry of Older South Australians (ROSA) is a crosssector multidisciplinary (ie, aged care and healthcare) platform built to address the challenges of monitoring people in aged care settings. This protocol describes the ROSA's framework and plans.

Methods and analysis A registry to capture 16000 South Australians/year undergoing an aged care eligibility assessment was designed. ROSA will contain information captured by the Commonwealth and South Australian state Health Authority, linked by two data integrating authorities, and housed on a secured data platform. ROSA will contain information on the sociodemographic, health, function, psychological, social, home and safety assessment and concerns characteristics, aged care services, general health services, and mortality of people receiving aged care services. Registered participants will be prospectively monitored until their death and yearly updates of their aged care and healthcare services information will be added to the registry.

Ethics and dissemination ROSA will Iongitudinally monitor the services provided to a population that puts costly demands on the state healthcare and aged care systems, identify unwanted variation, and underpin future research. ROSA's expected outputs include an annual report, a research agenda that focuses on high burden conditions and potentially economically impactfu questions, educational materials, and risk profiling tools. ROSA was approved by the South Australian Department for Health and Ageing HREC (HREC/17/SAH/125) and the Australian Institute of Health and Welfare HREC (E02018/2/429).

\section{INTRODUCTION}

Australia is an ageing country. ${ }^{1}$ In 2016, $15 \%$ (3.4 million) of Australians were over 65 years old and this proportion is expected to increase to $19 \%$ by $2031 .^{2}$ In the state of South Australia (SA), the proportion of people over 65 has already reached $18 \%$ and those over 85 are now more than $2.6 \%{ }^{3}$ of the

\section{Strengths and limitations of this study}

The Registry of Older South Australians (ROSA) is a unique data infrastructure with data from both the health and aged care sectors.

- Complete capture of the state-based population receiving subsidised aged care services, including longitudinal follow-up and minimal attrition of the cohort.

- By utilising only existing data ROSA minimises the burden on participants, eliminates the data collection process, demands and certain biases associated with collecting new data, while simultaneously maximising the capture of the cohort of interest and minimising selection bias.

- Our findings will be generalisable to all Australians seeking aged care services and could also influence the provision of aged care services to other states and countries with similar ageing populations placing increasing demand in the aged, health and social welfare sectors.

- A limitation of this cohort is that it only includes older adults accessing aged care services.

population, making it one of the states with the oldest population in the country. ${ }^{2}$ Nationally, $10 \%$ of Australians over 65 years old are receiving some type of aged care services at a cost of $\$ 18.1$ billion/year to the government alone. ${ }^{4}$ Additionally healthcare expenditure in Australia is over $\$ 170$ billion/year ${ }^{5}$ and older Australians are accountable for a significant proportion of this cost, since they are responsible for $41 \%$ of the hospitalisations nationally, $48 \%$ of the days spent in hospital, ${ }^{6}$ and use ambulance services at much higher rates than younger people. ${ }^{7}$ The demand on both the aged care and health services sectors will continue to increase with our ageing population, ${ }^{1}$ therefore evidence to support significant, efficient and cost-effective resource allocation is necessary.

Australians are fortunate to have aged care services, social services and a healthcare system subsidised by the government. Through 'My Aged Care' one can be assessed for aged care 
eligibility, which then results in likely support for aged care services by the Australian Commonwealth. ${ }^{8}$ Similarly, once registered with Medicare, one can obtain medical services and pharmaceuticals that are covered or partially subsidised by the government. To monitor the provision of these services, each government body has developed a data collection system, which individually monitors their areas of services delivered. ${ }^{9}$ Brought together these data sources have an incredible potential to comprehensively describe the ageing pathway and aged-related decline of older Australians, and could be the basis for creating the evidence necessary to support future allocation of resources for our ageing community.

The Registry of Older South Australians (ROSA) launched in April 2018 with the support of the South Australian state government and will conduct longitudinal evaluations of aged care recipients and their needs, both within the aged care services and health services domains, and evaluate the appropriateness and effectiveness of services and care to this population. This registry is the product of the Healthy Ageing Research Consortium, a cross-sectoral partnership of researchers, clinicians, aged care providers and consumer advocacy groups whose expertise encompasses geriatrics, gerontology, aged care services, general health and well-being. Specifically, the ROSA's main goals are to: (1) create an unique data infrastructure with cross-sector data-linkage, powerful analytical and hypothesis-testing capacity; (2) produce robust evidence for cost-effective service innovations, updated models of care, and other solutions for the ageing sector; (3) enable the monitoring of the quality of ageing in South Australia; (4) support and provide the infrastructure for a 'Living Lab'. ROSA's framework was developed with active support and involvement from consumer representative organisations and end users, which helped focus on activities that are of specific relevance to this population. With the infrastructure developed and leadership of ROSA we will be in a position to provide evidence to academics, health service providers, clinicians and the aged care industry. Furthermore, we also hope to influence future decisions by the government on the provision of aged care and health services to our ageing population.

This protocol describes the ROSA framework and plan.

\section{METHODS AND ANALYSIS}

The ROSA's framework adheres, where appropriate, to the 41 operating principles for clinical quality registries recommended by the Framework for Australian clinical quality registries. ${ }^{10}$ In summary, these principles define the need for a clinical quality registry to have a clear purpose and employ a methodologically sound design, appropriate data collection methods and approach, clearly specified data elements, risk adjustment capabilities, appropriate data quality monitoring, established organisation and governance, data custodianship agreements, appropriate ethics considerations, and clear output and publication plans. The ROSA target population, approach to data capture, data aggregation and storage, and expected output are described in this protocol and summarised in figure 1.

\section{Target population}

The target population of ROSA are South Australians who undergo an aged care eligibility assessment performed by an Aged Care Assessment Team (ACAT) member. The typical person having an assessment is an 84-year-old woman $(66 \%$ of total) with a one in two chance of having dementia. ${ }^{11}$ In the 2015/2016 financial year 15459 assessments were performed in South Australia. ${ }^{8}$ The aged care eligibility assessment confirms that the person being assessed is an older person who needs (1) coordinated services to help stay in their home; (2) to receive services through transition care; (3) to receive respite care in an aged care home; or (4) to move into an aged care home. ${ }^{12}$ While unlikely $(<4 \%$ of cases in 2015/2016) it is possible that a younger person with a disability, dementia or other special needs that are not met through other specialists services will be assessed for aged care services by an ACAT. ${ }^{11}$ These younger people will not be monitored by the registry at this time.

ROSA has initially targeted one ageing cohort, that is, South Australians assessed for aged care services for the first time, because it is a cohort with substantial information characterising them at specific time period. This cohort is also one with high health services utilisation and likely to obtain substantial benefit from the proposed body of work and importantly, it is also a feasible one at the scale ROSA is planned. We recognise that the findings of ROSA may have limited generalisability to other ageing cohorts because this cohort is of people accessing aged care services, and at this time there is limited evidence informing us to what degree the ROSA population is different from similar ageing cohorts. While aged care service preferences, laws, and the complexity of the case mix have changed dramatically in the last couple of decades ${ }^{13-15}$ as of $2016,70 \%$ of aged care 'places' were in residential care facilities, $28 \%$ were with home care packages and the remaining in restorative care. With the establishment of ROSA, a better understanding of this cohort and its limitations in regard to evaluating areas of ageing will be identified and will then be used as justification and support for enrolment of other cohorts into ROSA. It is the ROSA's objective to improve the quality of ageing for all Australians, therefore scaling ROSA to include other ageing cohorts is a future goal.

\section{Approach to data capture}

Entry into the ROSA will be triggered when an older South Australian undergoes an assessment by an ACAT member. ROSA is using an opt-out consent process to prospectively enrol participants. This was deemed acceptable after surveying a cohort of older Australians about acceptability of different consenting approaches, ${ }^{16}$ and was approved by the SA Health and the Australian Institute of Health and Welfare (AIHW) human research 


\section{Registry of Older South Australians (ROSA)}

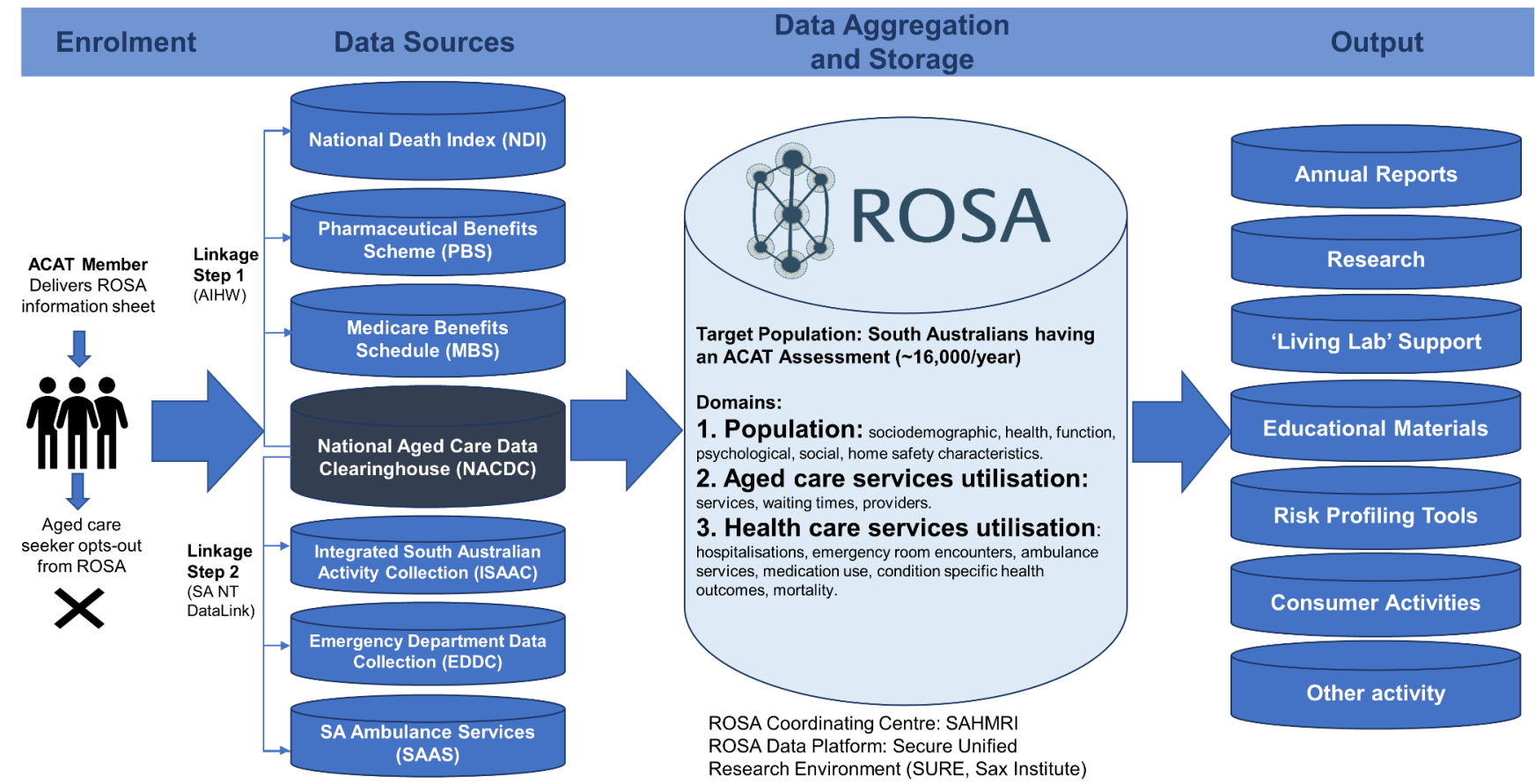

Figure 1 Framework for the Registry of Older South Australians (ROSA). ACAT, Aged Care Assessment Team; AlHW, Australian Institute of Health and Welfare; SAHMRI, South Australian Health and Medical Research Institute; SURE, Secure Unified Research Environment.

ethics committees. All prospective participants are provided with the ROSA Participant Information Sheet/ Opt-out Consent Form and either the participant or their guardian or carer, may choose to opt-out of ROSA at any time. ROSA launched its prospective enrolment in a staged fashion across South Australia's Local Health Networks starting in April 2018, and from early August 2018 it has been rolled out state-wide.

After enrolment into ROSA, the individual level participants' information will be obtained from the ACAT assessment itself, which is captured by the Commonwealth and extracts are housed in the National Aged Care Data Clearinghouse (NACDC), maintained by the AIHW. No other documentation will be required to be collected from the participant or ACAT member to minimise participation burden. ROSA will use the aged care assessment information captured in the NACDC to identify and characterise its cohort of interest and start its surveillance. In addition to the NACDC, ROSA will leverage existing Commonwealth and SA Health data sources to capture information on the aged care and healthcare services received by this cohort. Specifically, information from seven data sources, including the datasets from the NACDC, Medicare Benefits Schedule (MBS), Pharmaceutical Benefits Scheme (PBS), National Death Index (NDI), Integrated South Australian Activity Collection (ISAAC), Emergency Department Data Collection (EDDC) and SA Ambulance Services (SAAS), will be obtained for ROSA (figure 1). See table 1 for information on data source custodians and descriptions. Linkage for ROSA will occur annually and will be conducted by engaged data integrating authorities (ie, AIHW and SA NT DataLink).

Using the proposed datasets ROSA will construct variables that describe the following domains: sociodemographic characteristics, general health, function, physiological well-being, social welfare, home and safety assessment and concerns, medical history, aged care services approved and accessed, general health services utilisation, mortality, and other disease and aged care services specific outcomes. See table 2 for specific variables within the data domains that ROSA will capture. ROSA will monitor its registered cohort until the outcome of death. The NDI will provide ROSA with this outcome and reason for death. Less than $0.4 \%$ of the Australian population emigrated in 2012 with lower rates in older Australians, ${ }^{17} 18$ therefore we expect to have minimal lost to follow-up in the cohort given that $90 \%$ of ROSA's expected participants are over 70 years old. ${ }^{11}$

ROSA's framework is based on the concept that substantial information is already independently collected by government bodies in Australia. The ROSA decision to not collect any further information was a strategic one and is one of its strengths. By utilising only existing data, ROSA minimises the burden on the Registry participants, eliminates the data collection process, demands and certain biases associated with collecting new data, while maximising the capture of the cohort of interest and minimising selection bias. This model is not optimal for every registry, but it is supported in this instance where the current existing data covers the core data elements 
Table 1 Registry of Older South Australians' data sources, data custodians, data integrating authority, description of data source

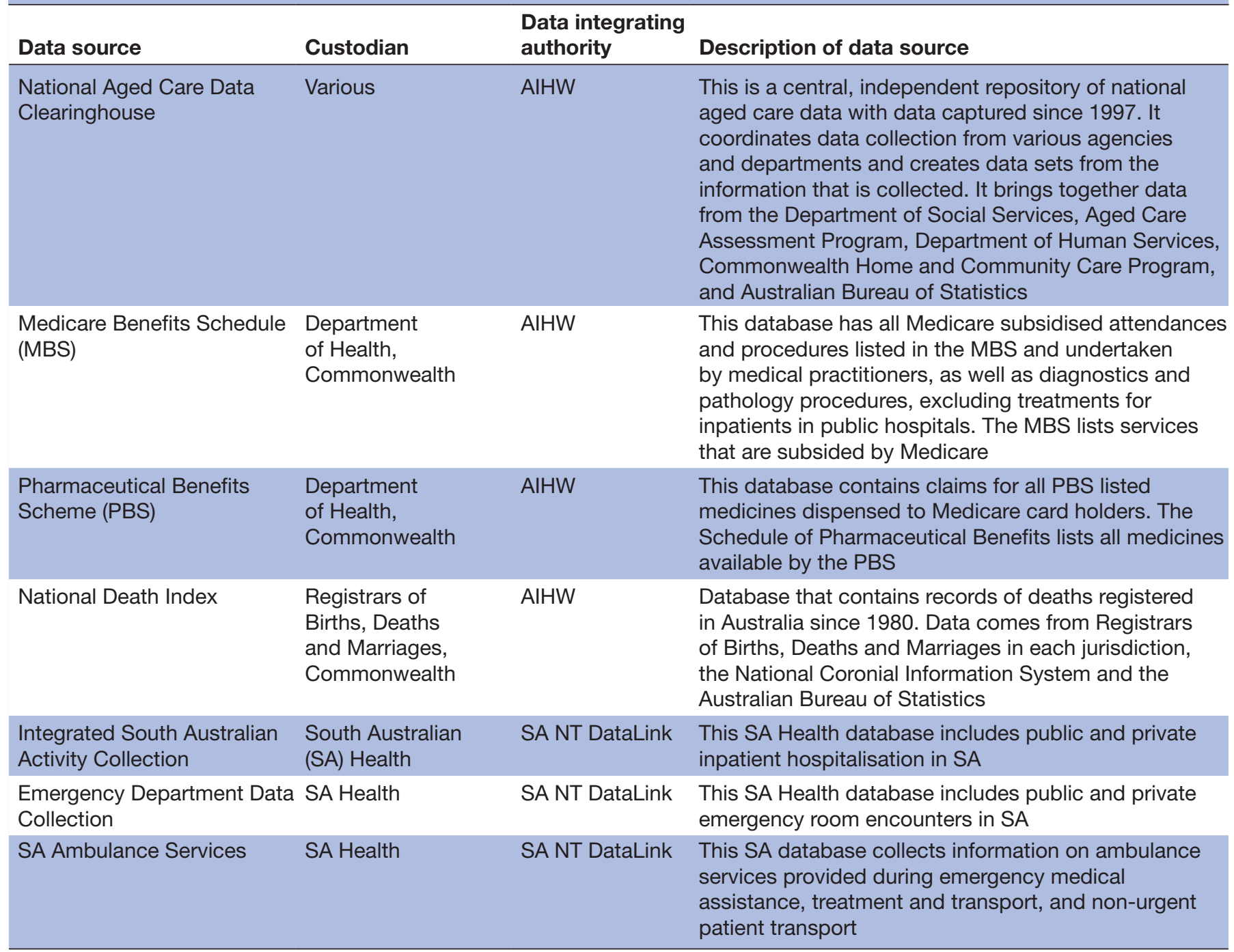

AlHW, Australian Institute of Health and Welfare.

necessary for the comprehensive description of our ageing cohort and the surveillance necessary to achieve the proposed aims of this project.

\section{Data storage and aggregation}

The ROSA coordinating centre is located at the South Australian Health and Medical Research Institute. Currently, ROSA is being designed to be hosted in the Secure Unified Research Environment (SURE), a secured and government approved data platform for housing datasets. ${ }^{19}$ In brief, SURE is housed at the Sax Institute in New South Wales and has security protocols that meet the challenges and privacy concerns of most government data custodians within Australia, making it the platform of choice for data linkage projects. SURE will provide a virtual workspace for the ROSA team, whereby the team will be able to create the database infrastructure and set up processes for data management and reporting using the SURE security protocols. ROSA is designed to be a relational database and SAS is the analytical tool of choice for data management and analysis by the ROSA team.

The ROSA data linkage will be led by the two data integrating authorities (AIHW and SA NT DataLink), and yearly extracts of the information identified as crucial will be requested. The first step in the linkage process is the identification of the cohort by the NACDC, which will be done by AIHW. After cohort identification, the cohort will be linked to their activity in the NDI, MBS and PBS databases. After this is complete the SA Health information, which includes the ISAAC, EDDC and SAAS activity are linked to ROSA by SA NT DataLink.

Once the data are made available to ROSA's analytical team, the data management process starts. This includes programming routines for data cleaning, which include quality control checks (including within and between cases logic), and outlier and missing data evaluation. After cleaning, the data will be processed to create yearly 
Table 2 Registry of Older South Australians' data domains and variables

\begin{tabular}{|c|c|}
\hline Domains & General variables \\
\hline Sociodemographic & $\begin{array}{l}\text { Age, gender, residency location, income level, marital status, country of birth, indigenous status, } \\
\text { pensioner status }\end{array}$ \\
\hline Health & $\begin{array}{l}\text { Medical history, comorbidities, frailty and other health concerns impacting on independence such as: } \\
\text { recent falls, pain, weight loss or nutritional concerns, feeling lonely, down or socially isolated, memory } \\
\text { loss or confusion, risks, hazards or safety concerns in the home, including environmental concerns and } \\
\text { special needs. When available Psychogeriatric Assessment Scales-Cognitive Impairment Scale score }\end{array}$ \\
\hline Function & $\begin{array}{l}\text { Ability/need for support in transport, shopping, meal preparation, housework, medicine management, } \\
\text { money management, walking, showering, dressing, eating, transfers, toilet use-assistance received, } \\
\text { assistance required. When available Transition Score (capability) on entry to service; functional capacity } \\
\text { is measured using the Modified Barthell Index }\end{array}$ \\
\hline Psychological & $\begin{array}{l}\text { Cognitive changes, such as changes in memory and thinking; changes in personality; behavioural } \\
\text { problems; psychological symptoms associated with memory loss, decision-making. Psychosocial } \\
\text { conditions or determinants such as feeling nervous, depressed or lonely, stressful events, changes in } \\
\text { mental state, and social isolation. When available Cornell Scale for Depression }\end{array}$ \\
\hline Social & Services and support currently being received, carer overview \\
\hline $\begin{array}{l}\text { Home safety } \\
\text { assessment }\end{array}$ & $\begin{array}{l}\text { Self-neglecting of care, equipment/modification required to maintain independence, personal and smoke } \\
\text { alarms; personal emergency plan, driving ability, concerns with living arrangements, concerns with } \\
\text { financial situation, safety concerns, legal issues }\end{array}$ \\
\hline Services provided & Care level, service type, start and end of services provided, service location, care pathways, payments \\
\hline Medication history & Dispensed medications and dispensing details \\
\hline Providers & Provider type, location \\
\hline General outcomes & Mortality, aged care services utilisation and changes \\
\hline Health outcomes & $\begin{array}{l}\text { Health services utilisation (ie, hospitalisations, readmissions to hospital after discharge, emergency room } \\
\text { encounters, general practitioners' visits, specialists' visits, ambulance services), quality indicators of } \\
\text { aged care services (ie, pressure ulcers, malnutrition), polypharmacy, inappropriate medication use and } \\
\text { other disease specific outcomes }\end{array}$ \\
\hline
\end{tabular}

ROSA files that include created/derived variables fitting the standard definitions created for ROSA's variables. These include variables such as comorbidity indices, frailty measures, counts of specific events, among others.

\section{Patient and public involvement}

The ROSA is the product of a partnership of 13 institutions, including two consumer advocacy organisations (Council on the Ageing SA and Health Consumers Alliance SA). These two organisations and seven consumer representatives are part of the governance structure of ROSA, which provided oversight for ROSA's development and now ongoing operations. Consumers were involved in the design of ROSA's Participant Information Sheet/Opt-out Consent Form, development of study proposals using ROSA's data, lay summaries of studies, and advise on opportunities for public and community engagements. In meetings scheduled four times a year, these consumers are updated on the progress of ROSA and findings from studies using the ROSA data, and have the opportunity to provide feedback.

\section{ETHICS AND DISSEMINATION \\ Ethics}

The Registry of Older South Australians (including the research protocol, data variables and participant information statement for opt-out consent) was approved by the South Australian Department for Health and Ageing Human Research Ethics Committee (HREC/17/ $\mathrm{SAH} / 125$ ) in March 2018, and by AIHW HREC (EO2018/2/429) in May 2018. This manuscript outlines the framework for the Registry of Older South Australians, it does not report any data collected from humans.

\section{Expected output and dissemination}

A ROSA annual report, which will include a comprehensive summary level (deidentified) description of the registered cohort, their overall aged care services utilisation, healthcare services utilisation, mortality, trends, and variation in these main areas will be prepared. These annual reports are intended to provide a cohort overview, general resource on the utilisation of aged care and healthcare services, and identify variations in need of further evaluation. These reports support ROSA's third objective, which is to monitor the quality of ageing in South Australia.

Other planned outputs include: translation of research findings into education materials for distribution at the point of contact with aged care recipients (aged care eligibility assessments, residential aged care setting, aged care providers), reports to support consumer activities (workshops/public lectures), reports to support and inform ACATs on their activities, and the development 
of risk profiling tools to support early identification of aged care recipients at risk of adverse events such as fractures, cardiac events and malnutrition. Additional reports requested by the ROSA Steering Committee or members of the Healthy Ageing Research Consortium may also be supported/provided on an ad-hoc basis.

The ROSA's full potential will be achieved through the utilisation of its information to create evidence for cost-effective innovations, updated models of care, and other solutions for the ageing and healthcare sector. ROSA's strategy to produce this evidence involves research focused within specific domains led initially by three post-doctoral researchers. These domains are: dementia and cognitive impairment, quality use of medicines, musculoskeletal health and health economics. These areas were chosen because they cover the most prevalent conditions in the ageing cohort and cover evaluations with relevant economic impact. Further involvement of honours students, PhD students, and researchers is also encouraged by the ROSA team. Engagement with partnering academics in opportunities for innovations is also a goal of ROSA. This includes the partnering in new research opportunities utilising ROSA data or in conjunction with ROSA to help achieve its goals.

Another key feature of ROSA is that it is designed to support a 'Living Lab'. This means ROSA provides the infrastructure and ability to support the trialling of innovative and emerging ideas to improve the health and wellbeing of ageing adults. Specifically, ROSA will be able to support 'nested' studies within the registry. ${ }^{20}$ With the proper administrative and ethics approval, ROSA can identify cohorts fitting specified criteria for studies and subsequently provide researchers with aggregate level data on the information captured by ROSA on these cohorts. This is an efficient and cost-saving model to conduct trials within the registry.

With a target enrolment of 16000 participants per year throughout the state of South Australia this is an ambitious effort, but one that is expected to provide a valuable resource that can be used to monitor the under-studied and not well understood population receiving aged care services in South Australia and their health services.

\section{Author affiliations}

${ }^{1}$ Registry of Older South Australians, South Australian Health and Medical Research Institute, Adelaide, South Australia, Australia

${ }^{2}$ Division of Health Sciences, University of South Australia, Adelaide, South Australia, Australia

${ }^{3}$ Discipline of Medicine, University of Adelaide, Adelaide, South Australia, Australia ${ }^{4}$ Rehabilitation, Aged and Extended Care, Flinders University, Adelaide, South Australia, Australia

${ }^{5}$ Division of Rehabilitation, Aged Care and Palliative Care, Southern Adelaide Local Health Network, Bedford Park, South Australia, Australia

${ }^{6}$ Helping Hand Aged Care, North Adelaide, South Australia, Australia

${ }^{7}$ National Health and Medical Research Council Centre of Research Excellence in Frailty and Healthy Ageing, University of Adelaide, Adelaide, South Australia, Australia ${ }^{8}$ Aged and Extended Care Services, Central Adelaide Local Health Network, The Queen Elizabeth Hospital, Woodville, South Australia, Australia

${ }^{9}$ Silver Chain Group, Keswick, South Australia, Australia

${ }^{10}$ South Australian Health and Medical Research Institute, Adelaide, South Australia, Australia
Acknowledgements We would like to acknowledge the Healthy Ageing Research Consortium Investigator Team, members of the Registry of Older South Australians (ROSA) governance committees and ROSA's South Australian Health and Medical Research Institute (SAHMRI) Research Team for ensuring the success of the ROSA and support with this study. We also acknowledge the South Australian Government who provide us with support through the Premier's Research and Industry Fund and the Department for Industry and Skills (2017-2021) to conduct this work, and the Australian Institute of Health and Welfare (AIHW) and SA Health for the provision of the raw data that will be used in ROSA. We would also like to acknowledge all the ROSA participants and the Aged Care Assessment Teams (ACAT) for their role in facilitating the enrolment of our cohort.

Contributors The following authors made substantial contributions to conception and design: $\mathrm{MCl}, \mathrm{SCEB}, \mathrm{CW}, \mathrm{MC}, \mathrm{RV}, \mathrm{KE}, \mathrm{ECG}$ and SLW. Acquisition of data: $\mathrm{MCl}$, SCEB, CW, ECG and SLW. Analysis and interpretation of data: MCI, SCEB, CW, MC, RV, KE, ECG and SLW. The manuscript was drafted by MCl and it was critically revised with input from SCEB, CW, MC, RV, KE, ECG and SLW.

Funding We acknowledge the South Australian Government who provide us with support through the Premier's Research and Industry Fund and the Department for Industry and Skills (2017-2021) to conduct this work.

Competing interests $\mathrm{RV}$ is on the board of Resthaven. KE represents an organisation (Silver Chain) that provides a Regional Assessment Service in South Australia and cares for individuals who have been ACAT Assessed. The remaining authors declare that they have no competing interests.

Patient consent for publication Not required.

Provenance and peer review Not commissioned; externally peer reviewed.

Open access This is an open access article distributed in accordance with the Creative Commons Attribution Non Commercial (CC BY-NC 4.0) license, which permits others to distribute, remix, adapt, build upon this work non-commercially, and license their derivative works on different terms, provided the original work is properly cited, appropriate credit is given, any changes made indicated, and the use is non-commercial. See: http://creativecommons.org/licenses/by-nc/4.0/.

\section{REFERENCES}

1. Australian Government. Productivity Commission 2013, An Ageing Australia: Preparing for the Future, Commission Research Paper, Canberra. https://www.pc.gov.au/research/completed/ ageingaustralia (Accessed 15 Aug 2017).

2. Australian Bureau of Statistics. Population Projections, Australia, 2012 (base) to 2101 (cat. no. 3222.0). 2013 https://www.abs. gov.au/AUSSTATS/abs@.nsf/Previousproducts/3222.0Main\% 20Features32012\%20(base)\%20to\%202101? opendocument\& tabname $=$ Summary\&prodno $=3222.0$ \&issue $=2012 \% 20$ (base) $\% 20$ to $\%$ 202101\&num=\&view = (Accessed 15 Aug 2017).

3. Australian Bureau of Statistics. Australian Demographic Statistics (cat. no. 3101.0). 2016 http://www.abs.gov.au/AUSSTATS/abs@.nsf/ DetailsPage/3101.0Dec\%202016?OpenDocument\#Time (Accessed 15 Aug 2017).

4. Australian Government Department of Health. 2017-18 Report on the Operation of the Aged Care Act 1997. Canberra. 2018 https://www. gen-agedcaredata.gov.au/Resources/Reports-and-publications/ 2018/November/2017\%E2\%80\%9318-Report-on-the-Operation-ofthe-Aged-Care-A (Accessed 24 Jan 2019).

5. Australian Government. Australian Institute of Health and Welfare. Health expenditure Australia 2015-16. 2017 https://www.aihw.gov. au/getmedia/3a34cf2c-c715-43a8-be44- 0cf53349fd9d/20592.pdf. aspx?inline=true (Accessed 24 Jan 2019).

6. Australian Institute of Health and Welfare. Admitted patient care 2015-16: Australian hospital statistics. Health services series no.75. Cat. no. HSE 185. Canberra: AlHW, 2017. https://www.aihw.gov. au/getmedia/3e1d7d7e-26d9-44fb-8549- aa30ccff100a/20742.pdf. aspx?inline=true (Accessed 13 Nov 2017).

7. Lowthian JA, Jolley DJ, Curtis AJ, et al. The challenges of population ageing: accelerating demand for emergency ambulance services by older patients, 1995-2015. Med J Aust 2011;194:574-8.

8. Commonwealth of Australia Department of Health. 2015-16 Report on the Operation of the Aged Care Act 1997. Canberra: ACT, 2016. https://www.gen-agedcaredata.gov.au/Resources/Reports-andpublications/2016/December/2015\%E2\%80\%9316-Report-on-theOperation-of-the-Aged-Care-A (Accessed 13 Nov 2017).

9. Davis J, Morgans A, Burgess S. Information management in the Australian aged care setting: An integrative review. Health Information Management Journal 2017;46:3-14. 
10. Australian Commission on Safety and Quality in Health Care. Framework for Australian clinical quality registries. Sydney: ACSQHC, 2014. https://www.safetyandquality.gov.au/wpcontent/ uploads/2014/09/Framework-for-Australian-Clinical-QualityRegistries.pdf (Accessed 1 Aug 2017).

11. Australian Institute of Health and Welfare. People Using Aged Care 2017. https://www.genagedcaredata. gov.au/Topics/People-usingaged-care/Explore-people-using-aged-care (Accessed 15 Jan 2018).

12. The Government of South Australia. SA Health. South Australian Aged Care Assessment Programme. Referral Pathways Protocol, 2016. http://www.sahealth.sa.gov.au/wps/wcm/connect/4ab0 930048ea8048bcecbff25a3eb7d6/ACAP+Re ferral+Pathways+ Protocol+V3+January+2016. pdf?MOD=AJPERES\&CACHEID= 4ab0930048ea8048bce cbff25a3eb7d6 (Accessed 12 Oct 2017).

13. Australian Government. Aged care act 1997. Compilation No. 66 . No. 112. 1997 https://www.legislation.gov.au/Details/C2017C00241 (Accessed 5 Sep 2017)

14. Australian Government, Department of Health. Ageing and aged care. Aged care reform 2016. https://agedcare.health.gov.au/reform/ aged-care-reforms (Accessed 15 Aug 2017).

15. Joenperä J, Van Der Zwan F, Karmel R, et al. Long and winding road: Aged care use before death. Australas J Ageing 2016;35:9-11.
16. Harrison SL, Milte R, Bradley C, et al. The acceptability of participating in data linkage research: research with older Australians. Aust N Z J Public Health 2018;42:497-8.

17. Australian Government. Department of Immigration and Border Protection. Fact sheet - emigration from Australia 2017. https:// archive.homeaffairs.gov.au/about/corporate/information/fact-sheets/ 05emigration (Accessed 11 Nov 2017).

18. Hugo G, Rudd D, Harris K. 2003. Australia's Diaspora: Its Size, Nature and Policy Implications. Final Report of an Australian Research Council (ARC) Linkage Grant conducted by the National Centre for Social Applications of GIS (GISCA), University of Adelaide; the Committee for Economic Development of Australia (CEDA) and the Department of Immigration and Multicultural and Indigenous Affairs (DIMIA). CEDA Information Paper No. 80.

19. Population Health Research Network. Sax Institute. Introduction to SURE 2017. https://www.saxinstitute.org.au/wp-content/uploads/ Intro-Guide-to-SURE-V3.0-Mar17.pdf (Accessed 12 Oct 2017).

20. Gliklich RE, Dreyer NA, eds. Registries for Evaluating Patient Outcomes: A User's Guide. 2nd edn. Rockville, MD: Agency for Healthcare Research and Quality, 2010. (Prepared by Outcome DEcIDE Center [Outcome Sciences, Inc. d/b/a Outcome] under Contract No. HHSA29020050035I TO3.) AHRQ Publication No.10EHC049. 